\title{
DETERMINAÇÃO EXPERIMENTAL DO EQUILÍBRIO LÍQUIDO-VAPOR DA MISTURA PSEUDO BINÁRIA BIODIESEL E ETANOL
}

P. M. S. KALVELAGE ${ }^{1}$, S. L. BERTOLI' ${ }^{2}$, A. A. C. BARROS ${ }^{3}$, E. L. SIMIONATTO ${ }^{4}$, L. P. FLORIANO $^{5}$

${ }^{1}$ Universidade Regional de Blumenau, Departamento de Engenharia Química

${ }^{2}$ Universidade Regional de Blumenau, Departamento de Engenharia Química

${ }^{3}$ Instituto Superior Politécnico de Tecnologias e Ciências, Departamento de Eng. e Tecnologias

${ }^{4}$ Universidade Regional de Blumenau, Departamento de Química

${ }^{5}$ Universidade Regional de Blumenau, Departamento de Engenharia Química

E-mail para contato: pollyanakalvelage@uol.com.br

RESUMO - A qualidade dos biocombustíveis está atrelada ao aprofundamento dos estudos sobre as diversas etapas dos processos de produção, principalmente as relacionadas com os processos de purificação. Para melhorar o desempenho desses processos, é necessário o conhecimento e a determinação do equilíbrio de fases que envolvem o equilíbrio líquidovapor. Os dados de equilíbrio de fases possibilitam estabelecer as condições adequadas de temperatura e pressão dos processos de separação presentes na recuperação do etanol ou metanol e na purificação do biodiesel, realizados por destilação, operação regida pelos princípios de equilíbrio entre as fases, líquido e vapor. Neste trabalho, são apresentados resultados experimentais do equilíbrio líquido-vapor da mistura pseudo-binária biodiesel e etanol, obtidos por técnicas de ebuliometria.

\section{INTRODUÇÃO}

Nos dias de hoje, os debates sobre os problemas ambientais passaram a ocupar lugar de destaque em todas as partes do mundo. É dentro desta perspectiva que muitos setores da sociedade moderna preocupam-se e conscientizam-se sobre a importância da preservação dos recursos naturais, principalmente quanto ao impacto do uso de combustíveis fósseis que, além de não serem renováveis, incorporam problemas ambientais.

Os biocombustíveis são alternativos aos combustíveis fósseis uma vez que a utilização destes promove a diminuição dos impactos ambientais. Os biocombustíveis mais conhecidos e utilizados na atualidade são o biodiesel e o bioetanol, cuja utilização é regulamentada no território brasileiro.

É com estas características que tem sido estudado e proposto o biodiesel como uma das alternativas aos combustíveis fósseis, pois é renovável e biodegradável, com emissões gasosas isentas de compostos sulfurosos, substâncias tóxicas e cancerígenas e emite $90 \%$ menos fumaça se comparado com o diesel mineral (ROCHA et al., 2008).

Para melhorar o desempenho dos processos de produção e purificação do biodiesel, requer-se a determinação das condições de equilíbrio de fases envolvida em cada etapa de separação dos processos referenciados, principalmente o equilíbrio líquido-vapor (ELV) presente 
na recuperação do etanol e purificação do biodiesel, realizados por destilação, operação regida pelos princípios de equilíbrio entre as fases líquido e vapor (COELHO, 2011).

O equilíbrio de fases e a modelagem termodinâmica aprofundam os conhecimentos dos processos químicos baseados em etapas que envolvem operações de separação industrial. Com base nestes princípios é possível conhecer e predizer o equilíbrio de fases necessário para avaliar o desempenho do processo no âmbito da eficiência de transferência de massa e calor e determinar as necessidades de utilidades do processo de produção e purificação (MEHL, 2009).

Nesta perspectiva, obtém-se dados do ELV para a mistura pseudo-binária etanolbiodiesel, conforme a metodologia a seguir descrita

\section{MATERIAIS E MÉTODOS}

\subsection{Materiais}

Para a execução deste trabalho foram utilizados os seguintes materiais: a) biodiesel; b) álcool etílico anidro PA; c) água destilada; d)óleo de soja e e)óleo de fritura utilizado no processamento de alimentos.

\subsection{Equipamentos}

Os equipamentos utilizados são os que seguem:

Micro Usina: O biodiesel utilizado neste estudo foi produzido utilizando-se a micro usina da produção de biodiesel projetada, construída e instalada no Departamento de Engenharia Química da Universidade Regional de Blumenau (FURB). A micro usina referenciada é uma unidade compacta, que incorpora diversas operações unitárias conforme a Figura 1.

Para a operação da micro usina, as seguintes etapas são realizadas:

a) Primeiramente, insere-se o óleo vegetal ou gordura animal no tanque (1) e do reagente (álcool) no tanque (2);

b) Em seguida, aciona-se a bomba (4), inferior, para que ocorra o transporte dos fluidos contidos nestes tanques para o tanque (5);

c) Concluído o transporte, aciona-se as resistências elétricas instaladas sobre a superfície do tubo (7) para iniciar o aquecimento da mistura contida no tanque (5);

d) Em seguida aciona-se a bomba (4), superior, para recirculação e aquecimento da mistura contida no tanque (5), onde a recirculação abrange o tanque (5), a bomba (4) e o tubo (7). O sistema é mantido aberto devido à presença do condensador (6) instalado sobre o tanque (5) e que garante a condensação e retorno do reagente vaporizado;

e) Depois de atingida a temperatura de reação, é inserido o catalisador já aquecido, no tanque (5), iniciando desta forma a reação de transesterificação;

f) Concluída a reação, incrementa-se a temperatura para recuperar o reagente (álcool) utilizado em excesso para garantir a reversibilidade da reação, abrindo desta forma a válvula de saída do álcool do condensador para o tanque (3);

g) Concluída a recuperação do álcool em excesso, a mistura remanescente é bombeada para o tanque (11), onde ocorre a separação das fases. Em seguida, utilizando-se a 
mesma bomba, direciona-se o biodiesel, fase leve, para o tanque (13) e o glicerol, fase pesada, para o tanque (12);

h) O biodiesel e o glicerol presentes nos tanques (12) e (13) passam por processos de purificação para garantir as especificidades comerciais;

i) O álcool em excesso recuperado e presente no tanque (3) também pode ser purificado, posteriormente, utilizando a coluna de destilação (9) acoplada ao "reboiler" (8). Os produtos desta etapa são coletados no tanque (10) e redirecionados para o tanque (2), voltando assim para o processo.

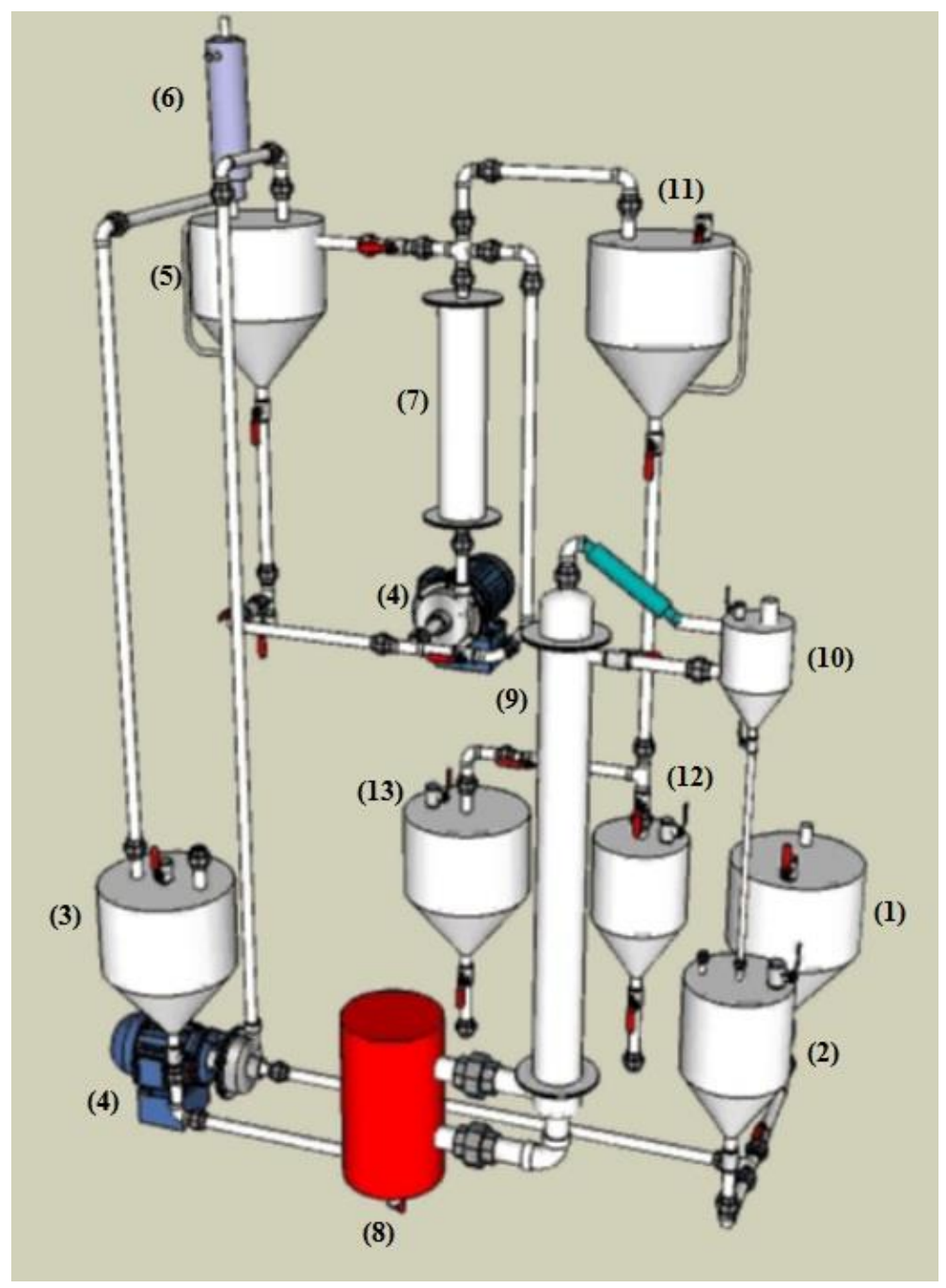

Figura 1 - Micro usina projetada, construída e instalada no Departamento de Engenharia Química da FURB e utilizada na produção do biodiesel.

Ebuliômetro Fischer: A Figura 2 mostra o ebuliômetro Fischer modelo 602, que consiste em uma célula dinâmica de medição de dados de equilíbrio líquido-vapor cujas fases, líquida e vapor, são circulantes. Este dispositivo é adequado para sistemas não eletrólitos e permite o 
estudo de substâncias com alto ponto de ebulição, desde que acompanhado com os devidos equipamentos de segurança. $\mathrm{O}$ ebuliômetro Fischer é um equipamento baseado na recirculação das fases líquida e vapor, em contato até o alcance do equilíbrio e é composto por: (1) frasco de mistura; (2) câmara; (3) tubo de ascensão da mistura ou 'bomba Cottrell"; (4) termopar; (5) condensador; (6) retirada de amostras da fase líquida e da fase vapor; (7) válvulas, todos conectados no sistema de ebuliometria.

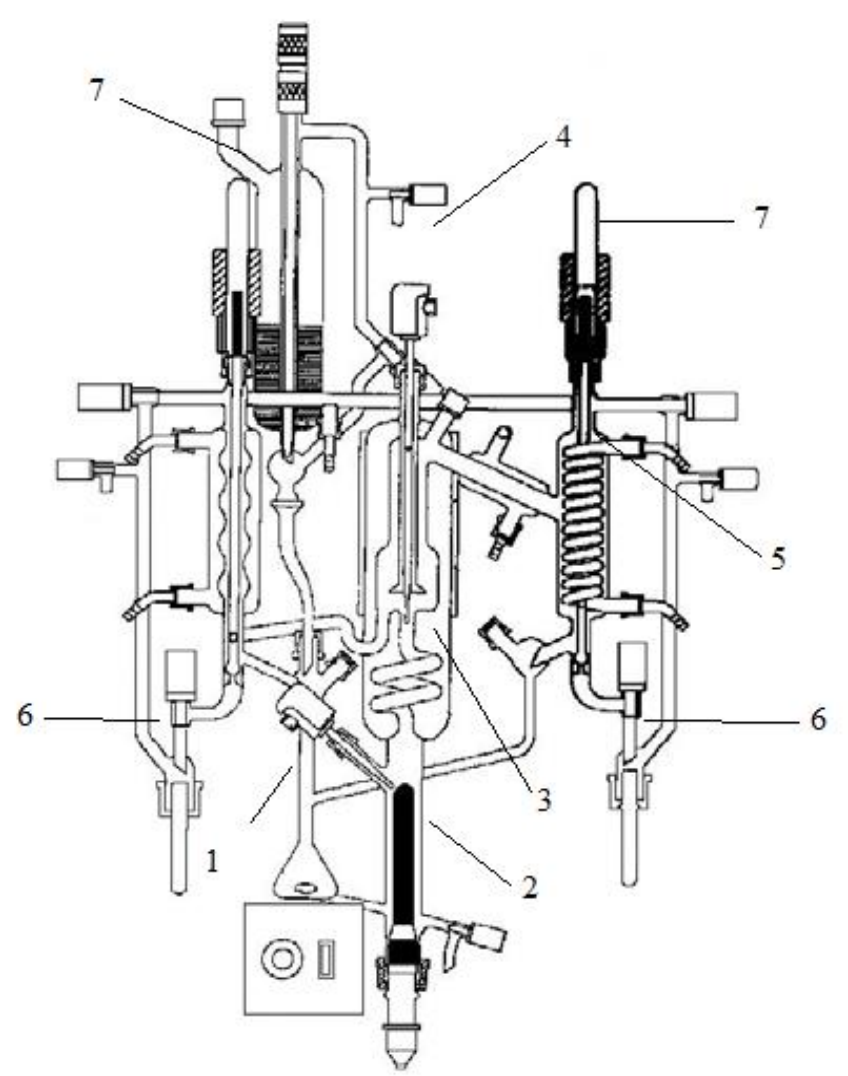

Figura 2 - Ebuliômetro de Fischer modelo 602 (Oliveira, 2003)

Cromatógrafo Gasoso: a análise de ésteres é realizada por cromatografia gasosa, que consiste em um método físico de separação dos componentes de uma mistura, que consiste na interação entre as fases formadas: a) uma fase permanece estacionária durante todo o processo; b) a outra fase move-se através da fase estacionária. Na cromatografia gasosa, a fase móvel é um gás e a fase estacionária é acondicionada na coluna, através da qual o gás de arraste flui. A amostra é introduzida na coluna através de um injetor, no qual o gás de arraste flui carregando a amostra. O componente da amostra cuja análise é feita, interage com as paredes da coluna da fase estacionária e cada componente flui em tempos diferentes, conhecido como tempo de retenção do composto que resultam na eficiência analítica da cromatografia gasosa.

Neste trabalho foi utilizado um cromatógrafo gasoso com detector de ionização por chama, modelo GC-2010 - SHIMADZU, com autoinjetor AOC-5000 de modelo headspace. 
Refratômetro: para a determinação do índice de refração da mistura etanol+água foi utilizado o refratômetro de abbe, onde a leitura é feita em uma escala graduada, através de um sistema ótico. neste caso, o valor medido é obtido mediante a comparação com uma tabela fornecida pelo fabricante do instrumento.

No refratômetro de Abbe se mede o ângulo limite da reflexão total, sendo que é possível se distinguir dois métodos de medição: um por transmissão onde a luz incide rasante, e outro por reflexão através da reflexão total.

\section{RESULTADOS E DISCUSSÃO}

\subsection{Análise do equilíbrio líquido-vapor da mistura pseudo binaria etanol-biodiesel}

A Figura 3 descreve o comportamento $\mathrm{T}-\mathrm{x}_{1}$ da mistura pseudo binária etanol-biodiesel, para o biodiesel obtido a partir do óleo de soja virgem. Os dados experimentais de equilíbrio líquido-vapor foram determinados operando-se com o sistema à pressão atmosférica em todos os ensaios realizados.

Os dados experimentais obtidos foram avaliados e comparados com os dados da literatura (GUO et al., 2007) mediante descrição gráfica da $\mathrm{T}-\mathrm{x}_{1}$, baseado nos princípios de equilíbrio líquido/vapor do sistema etanol (1)-biodiesel (2). No entanto, mesmo considerando a comparação feita, é fundamental registrar que os dados da literatura (GUO et al., 2007) foram obtidos experimentalmente a partir de pressões abaixo da pressão atmosférica, diferentemente da pressão utilizada neste trabalho. Por outro lado, o biodiesel utilizado no trabalho de Guo et al. (2007) foi obtido a partir do óleo de semente de girassol.

A comparação entre os dados da literatura e aqueles oriundos deste trabalho mostra a similaridade no comportamento destes dados principalmente para concentrações de etanol maiores que 50\%. Este comportamento é plausível dada a proximidade das pressões utilizadas nos dois processos aqui referenciados. É possível observar que os dados obtidos apesar da diferença de pressão de $6,32 \mathrm{kPa}$, praticamente sobrepõem-se entre si.

Na Figura 4 apresentam-se os dados T-x obtidos neste trabalho a pressão de 101,32 kPa e também os dados de Guo et al. (2007) na pressão de $95 \mathrm{kPa}$, para a mistura etanol e biodiesel obtido a partir de óleo de soja virgem e de óleo de semente de girassol respectivamente. Apesar da variação significativa de pressão de operação pode-se observar o comportamento qualitativo semelhante entre os dados.

As Figuras 3 e 5 também mostram similaridade de comportamento entre os dados obtidos neste experimento e os dados da literatura, apesar das diferentes pressões utilizadas e o biodiesel ser proveniente de diferentes fontes e serem produzidos por óleos em diferentes condições de operação. GUO et al (2007), utilizaram o biodiesel obtido a partir de semente de girassol a uma pressão de $95 \mathrm{kPa}$, enquanto que neste trabalho utilizou-se o biodiesel proveniente de óleo de soja virgem e a 101, $32 \mathrm{kPa}$. 


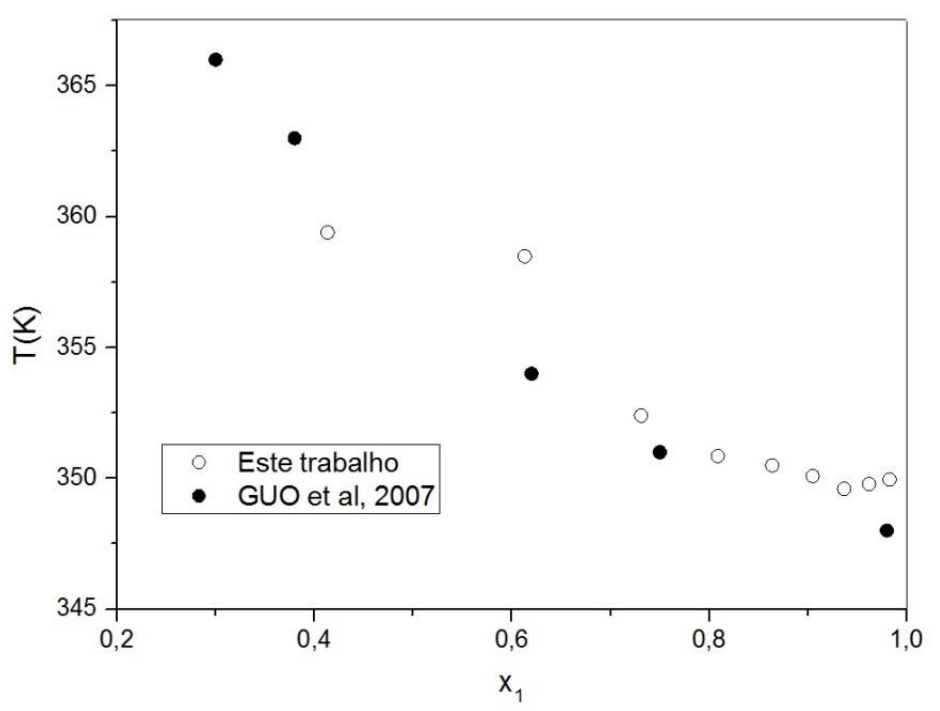

Figura 3 - Dados isobáricos ELV a 95kPa (GUO et al, 2007) e 101,32kPa (este trabalho) para o sistema binário etanol (1)-biodiesel(2) obtido a partir do óleo de soja virgem (este trabalho) e de sementes de girassol (GUO et al, 2007)

As Figuras 4 e 6 também demonstram similaridade, porém a pressão de trabalho dos dados da literatura é menor, $24 \mathrm{kPa}$. O biodiesel utilizado para a análise comparativa é proveniente da semente de girassol, e as condições experimentais do biodiesel a partir de óleo de soja (este trabalho) é de 101,32 kPa.

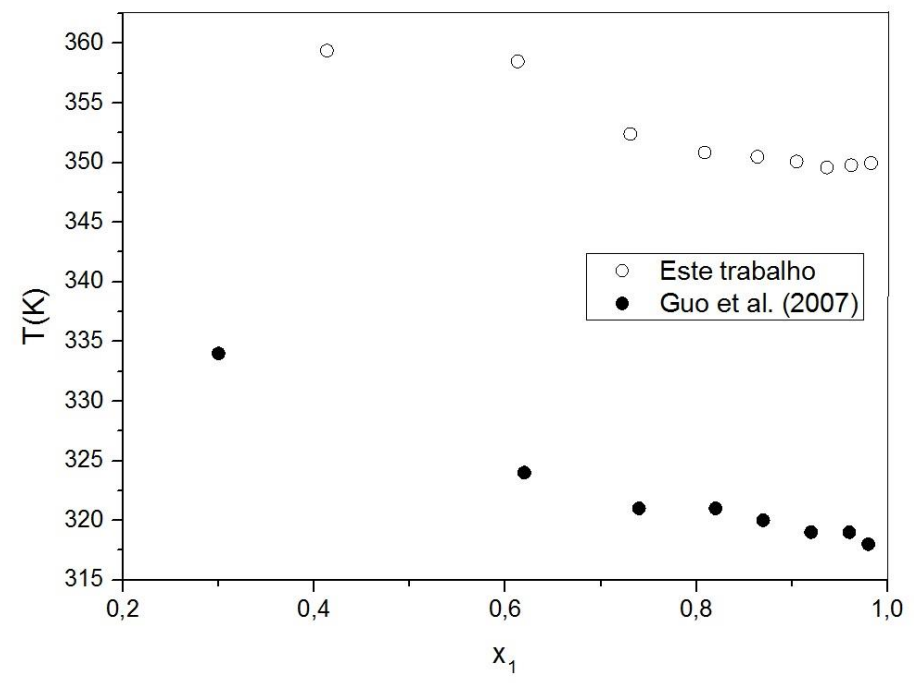

Figura 4 - Dados isobáricos do ELV a 24kPa (GUO et al, 2007) e 101,32kPa (este trabalho) para o sistema binário etanol (1)-biodiesel(2) obtido a partir do óleo de soja virgem (este trabalho) e do óleo da semente de girassol (GUO et al, 2007).

Nas Figuras 5 e 6 apresenta-se a comparação entre os dados obtidos a partir de biodiesel produzido com óleo de fritura utilizado no processamento de alimentos (este trabalho), com 
dados da literatura para biodiesel produzido a partir de óleo de girassol (GUO et al, 2007), sendo este último em pressão de $95 \mathrm{kPa}$ e $24 \mathrm{kPa}$, figuras 5 e 6 respectivamente. Desta forma pode-se afirmar que, similarmente ao óleo de soja virgem, o óleo de fritura e o óleo de semente de girassol estudados neste trabalho apresentam formação de ésteres de ácidos graxos com características físico-químicas similares, conforme as Figuras 5 e 6.

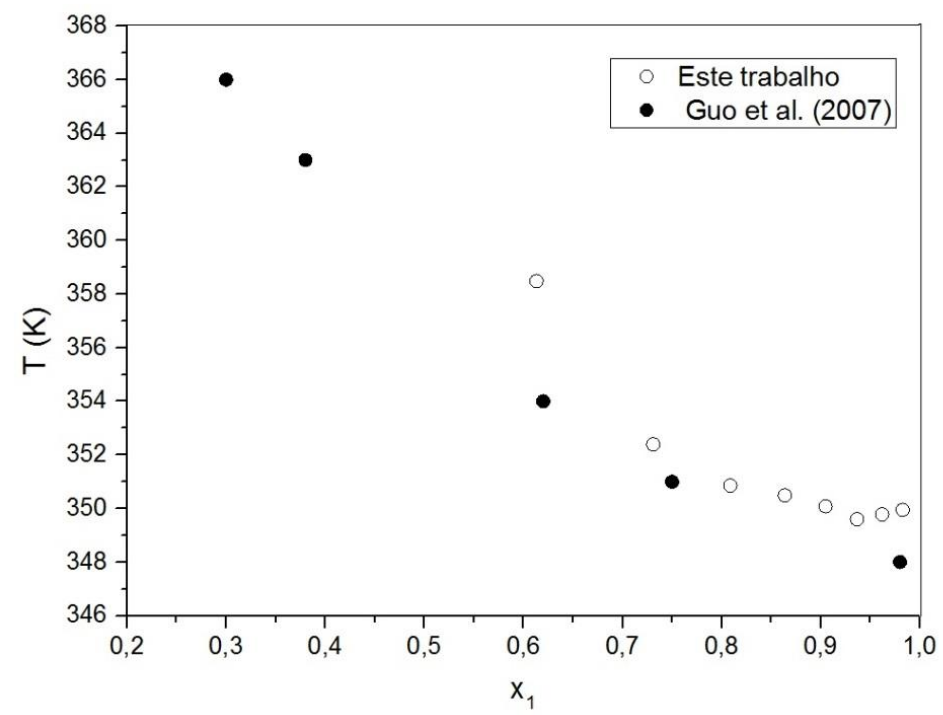

Figura 5 - Dados de isobárica do ELV a 95kPa (GUO et al, 2007) e 101,32kPa (este trabalho) para o sistema binário etanol(1)-biodiesel(2) obtido através do óleo de fritura (este trabalho) e do óleo de semente de girassol (GUO et al, 2007).

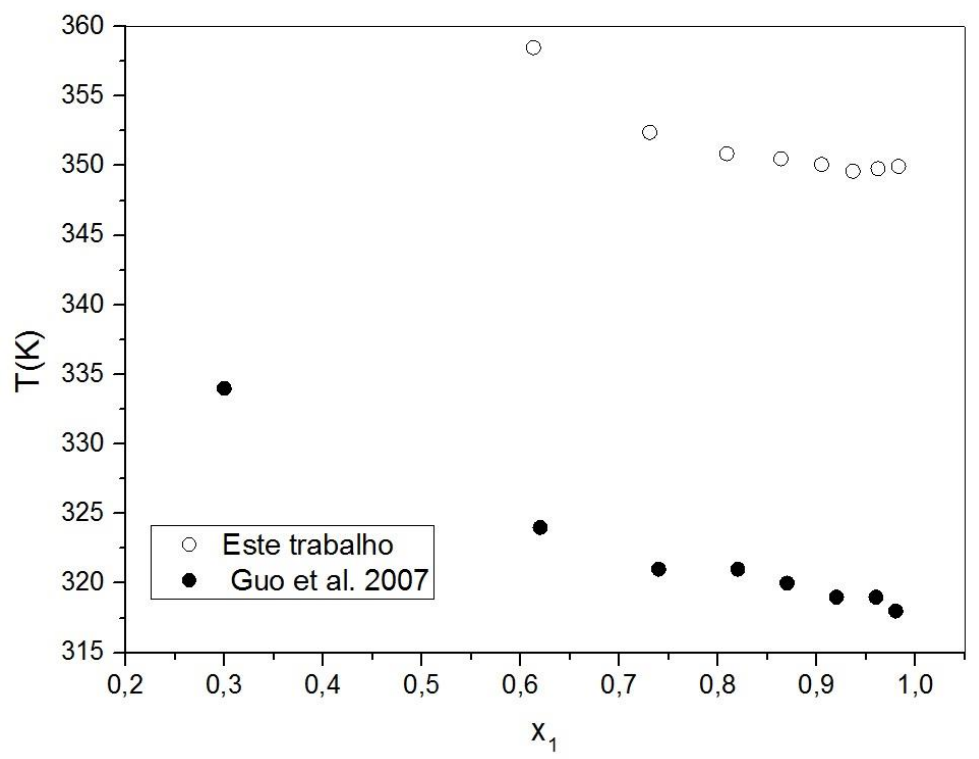

Figura 6 - Dados de isobárica do ELV a 24kPa (GUO et al, 2007) e 101,32kPa (este trabalho) para o sistema binário

Etanol(1)-Biodiesel(2) obtido através do óleo de fritura (este trabalho) e do óleo de semente de girassol (GUO et al,2007).

Conforme esperado, à medida que a fração de biodiesel aumenta, há uma tendência na elevação da temperatura de equilíbrio, tanto para a fase líquida quanto para a fase vapor. De 
acordo com o fabricante do ebuliômetro (ILUDEST DESTILLATIOSANLAGEM GMBH, 2011) temperaturas de ebulição maiores requerem acessórios especiais não disponíveis nessa unidade experimental, e por isso não foi realizada a aquisição de dados para frações de biodiesel próximas a 1. Além disso, este conjunto de dados apresentou dificuldade de regressão através do programa REGRESS. Isto se explica em função do limitado conjunto de pontos e da grande diferença de temperaturas de ebulição entre o biodiesel e o etanol.

\section{CONSIDERAÇÕES FINAIS}

I.O ebuliômetro do tipo Fischer, quando utilizado possibilita a aquisição de dados experimentais de equilíbrio líquido-vapor passíveis de comparação com dados da literatura e de uso nos projetos de construção dos equipamentos de separação;

II. A concordância dos dados experimentais da mistura binária etanol + biodiesel com os dados da literatura mostrou que, mesmo com diferenças de pressão e de matéria prima para a produção de biodiesel, o procedimento experimental e o ebuliômetro para o estudo de ELV de sistemas binários por componentes com grande diferença de volatilidade pode ser utilizado. Sugere também ser possível representar o comportamento termodinâmico do biodiesel a partir dos ésteres que o compõem, uma constatação importante para facilitar o estudo de tais sistemas em bancada experimental;

III.A falta de dados na região de concentrações de biodiesel elevadas dificulta a regressão dos mesmos através de modelos de coeficiente de atividade.

\section{REFERENCIAS}

COELHO, A. C. Equilíbrio Líquido-Vapor de Sistemas Binários Envolvendo Ésteres Etílicos do Biodiesel (Glicerol Ou Água) + Etanol: Dados Experimentais e Modelagem Termodinâmica. Impresso. Dissertação (mestrado) - Universidade Federal do Paraná, Setor de Tecnologia, Programa de Pós-graduação em Tecnologia de Alimentos, 2011.

GUO, Y. ; ZHONG, J. ; XING, Y.; LI, D.; LIN, R. Energy \& Fuels, v.21, p.1188 1192, 2007.

ILUDEST DESTILLATIOSANLAGEM GMBH. Installatio Manual for Equilibrium Apparatus Fischer Labodest VLE 602. Waldbuttelbrunn: Germany, 2011.125 p.

MEHL, A. Estudo do Equilíbrio de Fases de Hidrocarbonetos e $\mathrm{CO}_{2}$ Supercrítico. Tese de Doutorado em Tecnologia de Processos Químicos e Bioquímicos da Escola de Química da Universidade Federal do Rio de Janeiro. Rio de Janeiro, RJ, 2009.

OLIVEIRA, H. N. Determinação de dados de equilíbrio líquido-vapor para sistemas hidrocarbonetos e de uma nova célula dinâmica. 2003.162f. Tese (Doutorado em Engenharia Química). Universidade Federal do Rio Grande do Norte. Natal, RN, 2003. 
ROCHA, D. Q.; BARROS, D. K.; COSTA, E. J. C.; PASSOS, P. R.; VEIGA, V. F. V.; CHAAR, J. S. Determinação da material-prima utilizada na produção do biodiesel mineral através de monitoramento seletivo de íons. Quim. Nova, v. 31, n. 5, 1062-1066, 2008. 